\title{
Relaxed chromatin induced by histone deacetylase inhibitors improves the oligonucleotide-directed gene editing in plant cells
}

\author{
Hilda Tiricz ${ }^{1}$, Bettina Nagy ${ }^{1}$, Györgyi Ferenc ${ }^{1}$, Katalin Török ${ }^{1}$, István Nagy $^{2,3}$, Dénes \\ Dudits $^{1 *}$ and Ferhan Ayaydin ${ }^{1,4}$
}

\author{
${ }^{1}$ Institute of Plant Biology, Biological Research Centre, Hungarian Academy of Sciences, Szeged, Hungary \\ ${ }^{2}$ Institute of Biochemistry, Biological Research Centre, Hungarian Academy of Sciences, Szeged, Hungary \\ ${ }^{3}$ SeqOmics Biotechnology Ltd., Mórahalom, Hungary \\ ${ }^{4}$ Laboratory of Cellular Imaging, Biological Research Center, Hungarian Academy of Sciences, Szeged, Hungary
}

Corresponding author: Prof. D. Dudits

E-mail address: dudits.denes@brc.mta.hu

Institute of Plant Biology, Biological Research Center, Hungarian Academy of Sciences, Szeged, Hungary, Temesvári krt. 62, H-6726 Szeged, Hungary,

Tel: 003662 599-671, fax: $003662433-434$

\begin{abstract}
Improving efficiency of oligonucleotide-directed mutagenesis (ODM) is a prerequisite for wide application of this gene-editing approach in plant science and breeding. Here we have tested histone deacetylase inhibitor treatments for induction of relaxed chromatin and for increasing the efficiency of ODM in cultured maize cells. For phenotypic assay we produced transgenic maize cell lines expressing the non-functional Green Fluorescent Protein $(m G F P)$ gene carrying a TAG stop codon. These transgenic cells were bombarded with corrective oligonucleotide as editing reagent to recover GFP expression. Repair of green fluorescent protein function was monitored by confocal fluorescence microscopy and flow cytometry was used for quantification of correction events. Sequencing PCR fragments of the GFP gene from corrected cells indicated a nucleotide exchange in the stop codon (TAG) from $\mathrm{T}$ to $\mathrm{G}$ nucleotide that resulted in the restoration of GFP function. We show that pretreatment of maize cells with sodium butyrate $(5-10 \mathrm{mM})$ and nicotinamide $(1-5 \mathrm{mM})$ as known inhibitors of
\end{abstract}


histone deacetylases can cause elevated chromatin sensitivity to DNase I that was visualized in agarose gels and confirmed by the reduced presence of intact PCR template for the inserted exogenous $m G F P$ gene. Maize cells with more relaxed chromatin could serve as an improved recipient for targeted nucleotide exchange as indicated by an average of 2.67-3.62-fold increase in GFP-positive cells. Our results stimulate further studies on the role of the condition of the recipient cells in ODM and testing the application of chromatin modifying agents in other, programmable nuclease-based genome-editing techniques in higher plants.

Keywords: Gene targeting, Maize, GFP, Oligonucleotide, Chromatin.

\section{Introduction}

Increasing the precision in genetic engineering of plants is a central aim in plant sciences and crop improvement. For this purpose, several advanced technologies such as the use of zinc finger nucleases (ZFNs) and TAL effector nucleases (TALENs) as well as the CRISPR/Cas system can be considered as alternatives for the targeted editing of plant genomes (Puchta and Fauser 2014; Voytas 2013). As an additional option, synthetic chimeric RNA/DNA molecules or single-stranded DNA oligonucleotides (SDOs) can be employed as vectors to produce oligonucleotide-targeted nucleotide exchange (OTNE) at a specific site of plant genomic DNA (Breyer et al. 2009; Sauer et al. 2016a; Sauer et al. 2016b). The pioneering studies preferentially used chimeric RNA/DNA oligonucleotides for the induction of gene-specific mutations (Beetham et al. 1999; Kochevenko and Willmitzer 2003; Okuzaki and Toriyama 2004; Ruiter et al. 2003; Zhu et al. 2000; Zhu et al. 1999). Methodologies of oligonucleotide delivery into plant cells were based on either PEG-mediated uptake, alternatively on electroporation into tobacco, oilseed rape, Arabidopsis protoplasts (Kochevenko and Willmitzer 2003; Ruiter et al. 2003) or bombardment of oligonucleotide molecules co-precipitated onto gold particles into maize, oil seed rape, and rice cells (Beetham et al. 1999; Okuzaki and Toriyama 2004; Ruiter et al. 2003; Zhu et al. 2000; Zhu et al. 1999)

Oligonucleotide-directed mutagenesis $(\mathrm{ODM})$ as a precision genome-editing technology was successfully employed for the specific introduction of nucleotide exchange, and resulted in the synthesis of imidazolinone- or chlorsulfuron-insensitive forms of the aceto-hydroxyacid or acetolactate synthase (ALS) enzymes producing herbicide-resistant phenotypes (Kochevenko and Willmitzer 2003; Zhu et al. 2000). Despite these early results and an explosion in the adoption of various gene-editing tools, the ODM approach requires further improvement 
in reducing various limitations such as the low frequency of OTNE, the difficulty of selecting mutated cells, and the occurrence of off-target events. Out of several parameters, the optimal length and structure of oligonucleotide molecules need to be determined for a given organism. Sauer et al. (2016a) reported that oligonucleotide length had a positive correlation with the frequency of targeted edits as monitored by changing the blue fluorescent protein to green fluorescent protein. Dong et al. (2006) developed a transient assay system based on cobombardment of mutated GFP plasmid constructs with targeting SDO ${ }^{\mathrm{GFP}}$ to the scutellum cells of immature wheat embryos. In this experiment, the restoration of green fluorescence in the cells served as a visual marker for transient plasmid gene repair. The most common modification of oligonucleotides was phosphorothioate bond formation for their end-protection against exonucleases. It was frequently combined with modified structures such as locked nucleic acid (LNA) (Andrieu-Soler et al. 2005) or N-alkinylnucleotides (Fogarty et al. 2002) in order to increase the duplex-invading capability.

Development of OTNE as an efficient, large-scale methodology in plant gene editing requires the elucidation of underlying mechanisms. Our current knowledge on the basic mechanisms of OTNE originates from several studies performed exclusively on microorganisms and mammalian cells (Bonner and Kmiec 2009; Engstrom and Kmiec 2007; Sargent et al. 2011). According to the widely accepted general model, after invading the targeted site of the duplex DNA, SDOs are hybridized to the complementary strand through transient D-loop formation. Finally, the mutation is introduced into the DNA by cellular repair or replication machinery. One potential avenue for optimizing the efficiency of homologous exchange is through the induction of double-strand breaks (DSBs). This strategy was demonstrated in plants. Sauer et al. (2016b) reported that pretreatment of Arabidopsis protoplasts with the non-specific DSB reagent phleomycin improved OTNE frequency, furthermore the combined use of SDOs with engineered nucleases (TALENs or CRISPR/Cas9) allowed greater targeted genomeediting frequency. In these methods, the single-stranded DNA oligonucleotides serve as repair templates. Editing of the rice OSEPSPS gene was accomplished by co-transformation of TALEN with chimeric RNA/DNA oligonucleotides. By using SDOs in combination with the RNA-guided Cas9 endonuclease, the maize ALS2 gene was edited and chlorsulfuron-resistant plants were obtained (Svitashev et al. 2015). Furthermore, nucleotide change $(\mathrm{C} \rightarrow \mathrm{T})$ was built into the 5'-enolpyruvylshikimate-3-phosphate synthase (EPSPS) gene of flax that generated glyphosate resistance (Svitashev et al. 2015).

In mammalian cells DNA replication has also been shown to enhance the frequency of targeted gene repair presumably by destabilizing the chromatin structure (Brachman and Kmiec 2005; Hu et al. 2005; Huen et al. 
2006). Furthermore, the transcriptional activity of target genes can stimulate oligonucleotide-mediated gene repair (Huen et al. 2007). Several experimental findings call attention to chromatin modification and epigenetic reprogramming or the regulation of gene activity as potential targets in increasing the efficiency of OTNE methodology. The modulation of condensed and relaxed states of chromatin can be achieved by several mechanisms including DNA methylation or histone post-translational modifications such as acetylation. The presence of acetyl moieties on conserved lysines in the $N$-terminal extensions of nucleosomal core histones can neutralize the positive charge of lysines. This structure results in a lowered affinity of histones to the negatively charged DNA molecules and can cause chromatin relaxation and subsequent transcriptional activation. Histone deacetylation is catalyzed by histone deacetylases (HDACs). Inhibitors of HDACs are widely used to elucidate the developmental and physiological role of these enzymes that includes chromatin remodeling and transcriptional reprogramming (Delcuve et al. 2012). Studies on different plant tissues demonstrated that experimental induction of hyperacetylation of histones can be achieved by application of histone deacetylase inhibitors such as sodium butyrate (NaBt) or nicotinamide (NA) as shown by Earley et al. (2007) and Bond et al. (2009).

The cited studies provide sufficient basis to consider experimental chromatin modification with HDAC inhibitors as a testable approach to increase the frequency of gene editing in plant cells. For the present optimization of protocols for plant gene editing, we first generated an assay system that followed a strategy similar to that published earlier by Zhu et al. (1999). Using the vector construct with the mutated GFP gene from Dong et al. (2006) we produced transgenic maize cell lines and they were bombarded with synthetic GFP oligonucleotides for correction of the GFP function. Pretreatment of transgenic cells with NaBt or NA generated more relaxed chromatin in the region of the GFP transgenes and increased the potential for oligonucleotide-targeted nucleotide exchange. Here we discuss how the enhancement of oligonucleotide-mediated site-specific genomic modification can lay the foundation for the more extended use of this technology in the future.

\section{Materials and Methods}

\section{Maize cell culture and establishment of transgenic lines}

Zea mays (H1233) callus cells grown in suspension culture provided by Sándor Mórocz (Cereal Research Institute, Szeged, Hungary) were cultured in the presence of $0.5 \mathrm{mg} / \mathrm{L} 2,4$-dichlorophenoxyacetic acid (2,4-D) as described by Morocz et al. (1990). For treatment of maize cells with histone deacetylase inhibitors, the culture 
medium was supplemented with sodium butyrate (Sigma) or nicotinamide (Sigma) at final concentrations of 1 $\mathrm{mM}, 5 \mathrm{mM}$ and $10 \mathrm{mM}$. Cells were treated for $48 \mathrm{~h}$ prior to bombardment with the oligonucleotide.

The modified pAHC25 plasmid was used to generate a transgenic maize line with mutated Green Fluorescent Protein gene ( $m G F P$ ) (Dong et al. 2006). The original pAHC25 plasmid (Christensen and Quail 1996; Yao et al. 2006) was modified by removing the GUS gene using SacI and SmaI restriction enzymes, and the transformation plasmid pAHC25::mGFP was constructed by inserting the $m G F P$ gene between the SacI and SmaI restriction sites. Transgenic maize cells were produced using a biolistic transformation method by bombardment of the pAHC25::mGFP plasmid into the Zea mays (H1233) callus cell suspension. The transgenic cells were selected on N6M solid medium (0.2\% gelrite, Duchefa) supplemented with selective $20 \mathrm{mg} / \mathrm{L}$ L-phosphinothricin. Cells were cultured in darkness at $26^{\circ} \mathrm{C}$ for more than 2 weeks. Scarcely distributed PPT-resistant calli expected to be originated from single bombarded cells were picked up individually and grown further as separate clones. Clone 13 was used in the experiments.

The incorporated $m G F P$ gene was amplified from the extracted genomic DNA of putative transgenic lines using the CTAB method. PCR was performed with Thermo Scientific Phusion Hot Start II High-Fidelity DNA Polymerase. Amplified fragments with the expected size were recovered and sequenced directly in both directions (forward primer: 5'-GCTTGTGTCCGAGGATGTTT-3', reverse primer: 5'GGAGCAACCATGGGCAAGGGC-3').

\section{Synthesis of the correction oligonucleotide}

A single-stranded DNA oligonucleotide named SDO ${ }^{\mathrm{GFP}}$

(5'-ccaCCATGGTGAGCAAGGGCGAGGAGCTGTTCACCGGggtG-3'， lowercase letters stand for phosphorothioates) was used for correcting the mutation in the GFP gene (Dong et al. 2006). The synthesis of the $\mathrm{SDO}^{\mathrm{GFP}}$ molecule was performed using an Expedite 8909 DNA synthesizer (Applied Biosystems) by standard $\beta$-cyanoethyl phosphoramidite chemistry at a nominal scale of $0.2 \mu \mathrm{mol}$. The reagents for automated SDO synthesis were from Link Technologies, Sigma Aldrich, and Molar Chemicals Kft. SDO ${ }^{\text {GFP }}$ was purified by reverse phase HPLC.

\section{Bombardment of maize cells with plasmid and oligonucleotide molecules}

Cells and micro-colonies from maize suspension cultures were bombarded on filter paper (Whatman, grade 589/2, ashless) with plasmid pAHC25::mGFP $(5 \mu \mathrm{g})$ containing both $m G F P$ and $P A T$ genes for production of 
the stable transgenic lines. In correction experiments, the cells were bombarded with $5 \mu$ g single-stranded DNA oligonucleotides $\left(\mathrm{SDO}^{\mathrm{GFP}}\right)$ for nucleotide correction in the $m G F P$ gene as described by Dong et al. (2006).

\section{Fluorescence stereo microscopy and confocal laser scanning microscopy}

For fluorescence stereo microscopy screening, an Olympus SZX12 stereo microscope was used with GFP filter set. Confocal laser scanning microscopy was performed in an Olympus Fluoview FV1000 confocal laser scanning microscope. Microscope configuration was the following: objective lenses: UPLSAPO 20x (dry, NA: 0.75), UPLFLN 40x (oil, NA: 1.3); excitation lasers: $488 \mathrm{~nm}$ and $543 \mathrm{~nm}$; GFP and fluorescein diacetate (FDA) (1 $\mu \mathrm{g} / \mathrm{ml}, 5 \mathrm{~min})$ were detected between $505-530 \mathrm{~nm}$, propidium iodide (PI) $(1 \mu \mathrm{g} / \mathrm{ml}, 5 \mathrm{~min})$ was detected between 570-670 nm using spectral detectors. Transmitted light (bright field) images were captured with $488 \mathrm{~nm}$ laser line. Composite images were prepared using ImageJ software (National Institutes of Health, USA, version 1.41) and CorelDraw Graphics Suite X7 (Corel).

\section{Isolation of protoplasts and flow cytometry analysis}

Protoplasts were isolated from bombarded cell cultures according to Morocz et al. (1990).

The restoration efficiency of SDO ${ }^{\mathrm{GFP}}$ was measured by flow cytometry (BD FACSCalibur) of protoplasts from transgenic cells. In each sample at least $10^{5}$ protoplasts were analyzed. Background fluorescence was determined using non-transgenic cells as negative control. Flow cytometry measurement of samples was performed at $48 \mathrm{~h}$ post-bombardment.

\section{Analysis of GFP restoration events with Ion Torrent sequencing}

Following bombardment of control or $10 \mathrm{mM} \mathrm{NaBt-treated} \mathrm{maize} \mathrm{cells} \mathrm{with} \mathrm{SDO}{ }^{\mathrm{GFP}}$, single GFP-positive cells were picked up and placed into protoplast isolation solution using a dissection microscope. LabTek II coverslipbottomed 8-well chambers (Thermo Fisher) were used for overnight protoplastation. The enzyme was then removed by careful replacement with washing solution (WS) (2 x wash). Using a Leica DMI 6000 fluorescence microscope, GFP-positive protoplasts were located and marked in each chamber. Sparsely distributed GFPpositive single protoplasts were then manually picked up (in ca. $5 \mu 1$ volume) under sterile laminar flow using a standard automatic pipette and a simple inverted compound microscope. Protoplasts were pooled in $50 \mu 1 \mathrm{WS}$ solution in a microfuge tube on ice and frozen. Thawed and partially burst protoplasts were centrifuged in a benchtop microfuge for $5 \mathrm{~min}$ at $5000 \mathrm{~g}$ and WS was replaced with $100 \mu 1$ sterile distilled water. Burst protoplasts 
and nuclei were recentrifuged as before and resuspended in $20 \mu 1$ water. Samples were then vortexed (1 min) and boiled ( $3 \mathrm{~min}$ ) multiple times on a heating block for a total of 10 mins before sampling for PCR amplification.

Sequencing libraries were prepared by PCR where the product specific primers were flanked with either Ion P1 (5'-CCACTACGCCTCCGCTTTCCTCTCTATGGGCAGTCGGTGAT-3’) $\quad$ or $\quad$ barcode $\quad$ (BC80: 5'CCATCTCATCCCTGCGTGTCTCCGACTCAGTCGAAGGCAG

GCGAT-3') adaptors. PCR products were purified by using Agencourt AMPure XP beads (Beckman Coulter). Libraries were quality checked on D1000 ScreenTape and Reagents using TapeStation instrument (Agilent) and finally quantitated using Ion Library TaqMan Quantitation Kit (Life Technologies). The library templates were prepared for sequencing using the Life Technologies Ion OneTouch protocols and reagents. Briefly, library fragments were clonally amplified onto Ion Sphere Particles (ISPs) through emulsion PCR and then enriched for template-positive ISPs. More specifically, PGM emulsion PCR reactions utilized the Ion OneTouch 200 Template Kit (Life Technologies) and emulsions and amplification were generated using the Ion OneTouch System (Life Technologies). Enrichment was completed by selectively binding the ISPs containing amplified library fragments to streptavidin coated magnetic beads, removing empty ISPs through washing steps, and denaturing the library strands to allow for collection of the template-positive ISPs. For all reactions, these steps were accomplished using the Life Technologies ES module of the Ion OneTouch System. Template-positive ISPs were deposited onto the Ion chips (Life Technologies); finally, sequencing was performed with the Ion PGM Sequencing Kit (Life Technologies). The Ion PGM sequencing data were processed using CLC Genomics Workbench v8.5.1 (CLCBio); variant caller was programmed to run in high stringency mode requesting at least $12 \mathrm{X}$ read coverage and at least $66 \%$ mutation frequency. Only those variants were taken into account that were supported by sequencing on both strands; finally, variant regions were visually inspected.

\section{Analysis of chromatin accessibility by DNase I-PCR}

Preparation of nuclei: Nuclei were prepared (Paul and Ferl 1993; Paul and Ferl 1998; Shu et al. 2013) from 3 g filtered (Miracloth, Calbiochem) cell suspension on ice with $5 \mathrm{ml}$ of cold nuclei isolation buffer (NIB: $50 \mathrm{mM}$ Tris, $\mathrm{pH}$ 8.0, $0.3 \mathrm{M}$ sucrose, $5 \mathrm{mM} \mathrm{MgCl}_{2}, 10 \mathrm{mM} \beta$-mercaptoethanol, $1 \mathrm{X}$ protease inhibitor cocktail (Roche), $0.01 \mathrm{mM}$ phenylmethylsulfonyl fluoride (PMSF) and $0.1 \mathrm{mM}$ EGTA). NIB was added up to $25 \mathrm{ml}$ final volume and the suspension was incubated for $15 \mathrm{~min}$ at $4^{\circ} \mathrm{C}$ with gentle rotation. The suspension was filtered through 30 $\mu \mathrm{m}$ nylon mesh filters (Partec) and the eluate was centrifuged for $10 \mathrm{~min}$ at $1500 \mathrm{~g}$ at $4^{\circ} \mathrm{C}$. The supernatant was 
discarded, and the nuclear pellet was resuspended in $0.5 \mathrm{ml} \mathrm{NIB}$ as a crude nuclear extract. The DNase I digestion was carried out as previously described by Shu et al. (2013).

PCR analysis: PCR was performed using Red Hot Taq DNA Polymerase (Thermo Scientific) with primers for the ZmMEK1 and the $m G F P$ genes (ZmMEK1 forward 5'-GACCCCGGTAGACAAGTTCC-3', ZmMEK1 reverse 5'-GGTGACC

ATACACGGAATGC-3', $m G F P$ forward 5'-GTCTTGTAGTTCCCGTCATCCTT-3', $m G F P$ reverse 5'TGCAGTCGTAGGCGTTG-3'). In each reaction 50 ng DNA was used as template using 35 cycles. For PCR product quantification, the ImageJ Plot Analysis program was used.

\section{Statistical analysis}

For the statistical analyses at Figure 4, pairwise comparisons between control and inhibitor- treated samples were performed using Welch's t-test which is an adaptation of Student's t-test and is considered more reliable than Student's t-test especially if the samples have unequal variances (Ruxton 2006). Statistically significant changes using a confidence level of $95 \%(\mathrm{P}<0.05)$ were indicated with double asterisks. For statistical analyses, $\mathrm{R}$ statistical analysis software is used (developed by R Core Team, www.R-project.org/).

\section{Results}

Establishment of an assay system for detection of oligonucleotide-directed mutagenesis (ODM) in maize cells

The non-functional, mutated GFP gene $(m G F P)$ was reported by Dong et al. (2006) in which a $\mathrm{G}$ to $\mathrm{T}$ change was introduced creating a premature TAG stop codon. We used this gene variant to construct a maize transformation vector ( $\mathrm{pACH} 25)$ carrying the $m G F P$ gene under the control of the maize ubiquitin gene promoter. This vector also contains the selective phosphinothricin acetyltransferase marker $(P A T)$ gene under the control of the maize ubiquitin gene promoter. After the bombardment of cultured maize cells with this construct, we selected several independent phosphinothricin (PPT) resistant lines. Cell suspension culture was initiated from selected outgrowing resistant colonies and sequencing of PCR products of the $m G F P$ gene from genomic DNA confirmed the presence of the TAG sequence motif (Figure 1E). As expected GFP fluorescence signals could not be detected in these cells. 


\section{Restoration of GFP function by bombardment of transgenic maize cells with synthetic oligonucleotides}

Based on the known nucleotide sequence of the mutated $m G F P$ gene, oligonucleotide molecules were synthesized (see Materials and Methods). After bombardment of SDO ${ }^{\mathrm{GFP}}$ into maize cells, plates were screened using fluorescence stereo microscopy. Close-up images taken with laser scanning confocal microscope showed individual cells with bright green fluorescence (Figure 1A). After the restoration of GFP function, the maize cells were able to divide and form multicellular clusters (Figure 1B, C).

In addition to the microscopic observations, the frequency of GFP-positive cells was quantified by flow cytomerty. Under the used conditions, bombardments with corrective $\mathrm{SDO}^{\mathrm{GFP}}$ molecules led to an average of $0.0035 \%$ GFP-positive cells in several experiments. Majority of these correction events are assumed to be individual events, since samples were placed into cell wall digesting enzyme solution after 1 day post bombardment. Removal of cell walls halted progression of cell division cycle and resulted in flow cytometry analysis of primarily non-divided cells at $48 \mathrm{hrs}$ post bombardment. Moreover, liquid to solid medium change and bombardment-induced wounding stress also could play a role in retardation of divisions. We have observed first divisions of edited cells at around 60 hours post bombardment (Fig 1B). For sequence confirmation of OTNE, protoplasts were isolated from the GFP positive cells. The fluorescent protoplasts (at $48 \mathrm{~h}$ post-bombardment) were collected manually and used for DNA isolation (Figure 1D, see Materials and Methods). DNA fragments spanning the target site were amplified by PCR from the pooled DNA samples and were submitted to Ion Torrent analysis. Figure 1E presents the DNA sequence from the targeted mutant GFP gene that contains a stop codon (TAG) in place of the wild-type glutamic acid codon (GAG). Sequencing the corresponding PCR fragments from the corrected cells revealed that the majority of the reads contained the restored GAG codon (Figure 1E S1-S3). This change resulted in elimination of the stop codon and restored the green fluorescent phenotype. In addition to the expected nucleotide change, we also detected other nucleotide alterations although with low frequency (Figure 1E S2 and S3).

\section{Treatment of recipient maize cells with histone deacetylase inhibitors induces relaxed chromatin state}

For generation of open chromatin structure, we used sodium butyrate (NaBt) and nicotinamide (NA) as known inhibitors of histone deacetylase enzymes. The concentration range of these compounds applied in the present experiments was previously reported to induce an increased abundance of acetylated histone forms (Bond et al. 2009; Earley et al. 2007). The increased chromatin sensitivity to DNase I digestion is characteristic for the open chromatin state. This assay can be used to probe DNA accessibility at the whole genome level. Figure 2A shows total nuclear DNA electrophoresed on an agarose gel after treatment of inhibitor-exposed (or control) cells' 
nuclear DNA with various concentrations of DNase I, which caused DNA fragmentation depending on the level of chromatin accessibility. The thick, dark, high molecular weight bands are still visible in the control cell samples even after elevated concentrations of DNase I, while for the HDAC inhibitor-treated samples, the formation of smaller fragments are enhanced with increased amounts of DNase I used. This assay shows that more relaxed chromatin can be achieved by treatment with $5 \mathrm{mM}$ NA and $10 \mathrm{mM} \mathrm{NaBt}$ in maize cells.

Shu et al. (2013) reported a protocol for the use of DNase I-Polymerase Chain Reaction assay that provides chromatin accessibility information in selected genomic regions. In the present study, we used this technique to analyze the accessibility of the target $m G F P$ transgene. We also included the maize MAPKK gene $Z m M E K 1$ as alternative reference. Following the protocol described by Shu et al. (2013), we quantified the PCR products from both the $m G F P$ and the ZmMEK1 genes after digestion of nuclear DNA with different doses of DNase I enzyme. The principle of this approach is that the DNA of more accessible chromatin is cleaved with greater efficiency which results in increased fragmentation of DNA. Therefore reduced amounts of PCR product will be detected as compared to that of inaccessible chromatin. As shown in Figure 2B-D, after gel separation of PCR products, we quantified gel intensities using ImageJ Plot Analysis program. In testing the chromatin structure surrounding the mGFP gene, NaBt treatment caused significant reduction in PCR products after treatment with the highest concentrations of DNaseI as compared to undigested samples $(93.7 \%, 1 \mathrm{U}$ treatment; $87.3 \% 0.5 \mathrm{U}$ treatment). In the case of NA treatment, these reductions were more moderate $(40.8 \%, 1 \mathrm{U}$ treatment; $29.1 \%$ $0.5 \mathrm{U}$ treatment). In contrast $\mathrm{ZmMEK} 1$ gene products reflected higher reduction levels with similar trends after treatment with both inhibitor compounds. For samples without inhibitor treatments, elevated level of PCR products were detected which reflects less effective digestion with DNaseI enzyme. From these data we can conclude that exposure of maize cells to HDAC inhibitors can lead to open chromatin structure including the region of the target gene.

To assess the possible toxicity, we also tested the effect of these inhibitors on the viability of maize cell cultures using a two-color fluorescence viability assay (Figure 3). Fluorescein diacetate (FDA) is a non-fluorescent, membrane-permeable agent. When the acetyl groups of FDA are cleaved by cellular esterases, a green fluorescent "fluorescein" molecule is released inside live cells. Propidium iodide (PI), on the other hand, is a red fluorescent dye which enters only the membrane-compromised, dead or dying cells and emits red fluorescence after binding to cellular nucleic acids. Therefore the co-incubation of cells with FDA/PI can be used as a convenient dual-color viability assay. As compared to untreated control samples, treatment with 10 mM NA or $\mathrm{NaBt}$ for 48 hours did not cause a drastic reduction in viability as evidenced by the FDA/PI fluorescence 
viability assay. These studies showed that the exposure of maize cells to HDAC inhibitors can lead to open chromatin structure including regions around the target gene without causing significant toxicity problems.

\section{Pretreatment of maize cells with histone deacetylase inhibitors increased the frequency of cells with corrected GFP function}

To analyze the link between chromatin structure and the efficiency of ODM in plant cells, we tested the effect of chromatin-remodeling compounds that can induce a relaxed state of chromatin. Different concentrations of sodium butyrate $(\mathrm{NaBt})$ and nicotinamide $(\mathrm{NA})$ were used for the pretreatment of maize cells for $48 \mathrm{~h}$. When the

control, non-treated cell population was bombarded with correction $\mathrm{SDO}^{\mathrm{GFP}}$ molecules, the frequency of GFPpositive cells was calculated to be $0.0035 \%$ in average. The number of SDO-corrected, GFP positive protoplasts was calculated on the basis of flow cytometry analysis. Figure 4 shows that pretreatments with 5 and $10 \mathrm{mM}$ $\mathrm{NaBt}$ resulted in improved correction efficiency as compared to untreated control cells and resulted in a 2.38 and 3.62 fold increase, respectively though statistically significant only for the latter concentration. Treatment with 1 $\mathrm{mM}$ and $5 \mathrm{mM}$ NA caused statistically significant, 2.85 and 2.67 fold increase in the number of GFP-positive protoplasts, respectively. Using a higher, $10 \mathrm{mM}$ concentration did not further improve the efficiency, indicating a possible negative effect of this potent inhibitor when used at non-optimal concentrations.

\section{Discussion}

Although gene technology and conventional transgenesis have become integrated components in plant research and crop improvement, we can see growing interest in the use of efficient and widely applicable gene/genomeediting methods in plant sciences. Development of tools for designing programmable nucleases that introduce site-specific double-strand breaks (DSBs) for specific and effective genome editing and for oligonucleotidedirected mutagenesis (ODM) is in the focus of ongoing editing research and provides novel therapeutic opportunities and breeding applications (Gilles and Averof 2014; Kim and Kim 2014). One of the obstacles in achieving ODM with improved efficiency has been the optimization of the state of recipient cells. Here we tested how the chemical induction of open chromatin structure can influence the frequency of OTNE in cultured maize cells after bombardment with correction oligonucleotide molecules.

First we had to establish a test system for monitoring OTNE events in maize cells. For this purpose the restoration of green fluorescence function of a mutated, non-functional GFP gene can serve as a phenotypic 
indicator in the quantification of gene repair frequency, as has been demonstrated in different eukaryotic cell systems including plants (Engstrom and Kmiec 2008; Parekh-Olmedo et al. 2003). A transient assay system was reported by Dong et al. (2006) where cultured scutellum cells of immature wheat embryos were co-bombarded with a GFP gene carrying a stop codon mutation and correcting $\mathrm{SDO}^{\mathrm{GFP}}$ molecules. We present data where we compare efficiency of various treatment combinations using untreated control cells as reference (Figure 4). Following $10 \mathrm{mM} \mathrm{NaBt}$ treatment, the number of SDO-corrected protoplasts calculated on the basis of flow cytometric data was found to be 3.62 times more as compared to untreated control samples. This improvement is still limited, but considering the general low efficiencies in plant studies it can be considered as an advance in further development of the technology. It is rather difficult to compare these frequency data with those obtained in other experimental systems due to differences in methods applied. In an essentially different experimental system, Sauer et al. (2016b) treated protoplasts from transgenic Arabidopsis plants with a stably integrated blue fluorescent protein gene that was converted to a green fluorescent protein gene by PEG-induced uptake of oligonucleotides. In this case, less than $0.01 \%$ of the total protoplast population showed correction events with blue fluorescent signal.

The phenotypic readout of ODM was further supported by detection of the change in the stop codon (TAG) from $\mathrm{T}$ to $\mathrm{G}$ in the targeted gene region. As shown by Figure 1E (S1) the GAG triplet frequency was observed in high frequency both for control, non-treated cells and for NaBt-treated cells. It is reported in a recent review by Rivera-Torres and Kmiec (2016) that there are only very limited experimental data originating from plant research that can contribute to elucidating the mechanism of ODM. The concept of SDO-directed gene editing is primarily derived from studies on model systems using yeast cells or mammalian cell lines. The proposed mechanism includes the alignment of SDO with a complementary sequence of the target gene in the chromosome that generates a single mismatched base pair with the nucleotide targeted for change. After pairing, the SDO is hybridized to one strand of the helix, while the other strand is displaced forming a structure known as a D-loop. The distortion of the helical structure activates members of the homologous recombination repair pathway. Postulating similar molecular events in synthetic SDO-treated plant cells, one can define several factors to be optimized in the OTNE methodology for plants. In the present work we show that the chemically induced open chromatin structure in maize cells can support ODM as indicated by an increased frequency of restoration of mutant GFP phenotype after bombardment with correction oligonucleotide molecules (Figure 4).

Modification of chromatin structure can be achieved by experimental induction of the hyperacetylation of histones through application of histone deacetylase inhibitors. For this purpose, we selected sodium butyrate 
$(\mathrm{NaBt})$ and nicotinamide (NA) with effective concentrations known from published data. Treatment of tobacco seedlings for 6-24 h with $10 \mathrm{mM}$ sodium butyrate $(\mathrm{NaBt})$ or $10 \mu \mathrm{M}$ trichostatin (TSA) increased the acetylation state of histones H3 and H4, and activated the expression of PetE promoter (Chua et al. 2003). The treatment of Brassica oleracea (broccoli) inflorescences with $10 \mathrm{mM} \mathrm{NaBt}$ for $36 \mathrm{~h}$ increased the abundance of acetylated histone H4 isoforms (Earley et al. 2007). Treatment of Arabidopsis seedlings with $5 \mathrm{mM}$ nicotinamide (NA) for 14 days produced an approximately twofold increase in the level of histone $\mathrm{H} 3$ acetylation within the coding region of VERNALIZATION INSENSITIVE 3 (VIN3) (Bond et al. 2009). In their study all three HDAC inhibitors (NaBt: $2 \mathrm{mM}$; TSA: $5 \mu \mathrm{g} / \mu \mathrm{l}$; NA $5 \mathrm{mM}$ ) induced an enhanced expression of the VIN3 gene. In our experiments, even the highest $(10 \mathrm{mM})$ concentration of these inhibitors displayed comparable viabilities to control cultures as judged by dual color fluorescence live-dead staining assay (Figure 3).

Our data presented here provides experimental evidence for the induction of relaxed chromatin by the treatment of plant cells with HDAC inhibitors as epigenetic modifiers. The increased DNase I sensitivity of nuclei isolated from NaBt- and NA-treated maize cells was demonstrated by resolving digestion products on agarose gel (Figure 2A). This reflects the enhancement of the general accessibility of chromatin at the whole genome level. The frequency of OTNE events is expected to be dependent on chromatin organization at the loci of transgenic mGFP gene. Using DNase I-Polymerase Chain Reaction Assay (Shu et al. 2013), we show enhanced chromatin accessibility induced by HDAC inhibitors in the chromosomal region where the $m G F P$ gene is located. Both DNase I assays (Figure 2A, B) indicate stronger chromatin modifying effects for NaBt than in the case of NA at the applied concentrations. As it can be seen in Fig 2C and 2D, using undigested DNA from the inhibitor treated cells, the PCR reactions were less efficient as compared to DNA samples from control (inhibitor untreated) cells. In addition to the epigenetic effect on histones, both $\mathrm{NaBt}$ and $\mathrm{NA}$ can generate complex cellular changes including activation and repression of set of genes. Therefore, it is possible that reduced amplification response may arise from the presence of elevated levels of unknown PCR interfering compounds in NaBt and NA-treated cells. Compounds such as polyphenols, pectins, polysaccharides and xylan are known to be inhibitory to plant PCR reactions as reviewed by Schrader et al. (2012). As shown in Figure 4, both agents effectively increased the frequency of GFP-positive cells as indicators of gene repair events. The responses were concentration dependent, and the treatment with $10 \mathrm{mM} \mathrm{NaBt}$ resulted in maximum effect. In the case of NA treatment, concentrations of 1-5 $\mathrm{mM}$ caused a significant increase in efficiency. The higher frequency of correction events in the NaBttreated cells can be correlated with the elevated DNase I sensitivity (Figure 2). The 2.83-3.62-fold increase in 
ODM in plant cells is in the range of efficiency improvement caused by trichostatin (TSA), which is used for HDAC inhibition in yeast and HeLa cells (Parekh-Olmedo et al. 2003).

In the present work using maize cells, the experimental design was focused on the relation between chromatin structure and ODM events. Both $\mathrm{NaBt}$ and NA treatments were active in generation of relaxed chromatin and increasing the frequency of GFP positive cells. Due to scarcity of gene editing information regarding plant cells, it is difficult to predict an exact molecular basis of the observed findings. However, it is tempting to speculate that the induction of relaxed chromatin primarily accounts for the alteration of the frequency of ODM events in our experimental system. Our data may initiate further studies for better understanding factors determining efficiency of oligonucleotide-directed gene editing in plant systems.

Genome engineering with OTNE shares common molecular mechanisms with the repair of targeted doublestrand breaks induced by engineered sequence-specific nucleases. In both cases the sequence recognition and activation of cellular repair pathways are expected to be dependent on chromatin organization. On this basis, chromatin modifying agents can be instrumental to elevate the efficiency of gene editing using zinc finger nucleases (ZFNs), TAL effector nucleases (TALENs) and the CRISPR/Cas system in plants. An advanced OTNE technology can be a key component in the combinatorial use of oligonucleotides with programmable nucleases. In human and mouse cells the expression of either ZFNs or TALENs in combination with SDO stimulated OTNE-mediated gene repair (Radecke et al. 2010; Strouse et al. 2014; Wefers et al. 2013). Recent publications provide further examples for plant gene editing by co-transformation of SDO molecules and sequence-specific nucleases (Svitashev et al. 2015; Wang et al. 2015).

By offering alternatives to traditional transgenesis, the progress in the improvement of these genomeediting technologies is of central significance as novel breeding techniques (Breyer et al. 2009). Crops produced by these methodologies might have a greater acceptance by the GMO-conscious public, organizations and governments (Voytas and Gao 2014).

\section{Acknowledgments}

The authors thank Dr. Chongmei Dong (Plant Breeding Institute, University of Sydney, Australia) for providing the GFP vector constructs and Sándor Mórocz (Cereal Research Institute, Szeged, Hungary) for making the maize cell suspension culture available for transformation and Edit Kotogány for help with flow cytometry analyses. This publication is dedicated to the memory of the late Dr. Sándor Bottka, who inspired the use of 
synthetic oligonucleotides in our plant research. This work was supported by the National Research, Development and Innovation Office, NKFIH (Grant Number K116318 to FA) and János Bolyai Research Scholarship of the Hungarian Academy of Sciences (to IN).

\section{Conflicts of interest}

The authors declare that they have no conflicts of interest.

\section{References}

Andrieu-Soler C et al. (2005) Stable transmission of targeted gene modification using single-stranded oligonucleotides with flanking LNAs Nucleic Acids Res 33:3733-3742 doi:10.1093/nar/gki686

Beetham PR, Kipp PB, Sawycky XL, Arntzen CJ, May GD (1999) A tool for functional plant genomics: chimeric RNA/DNA oligonucleotides cause in vivo gene-specific mutations Proc Natl Acad Sci U S A 96:8774-8778

Bond DM, Dennis ES, Pogson BJ, Finnegan EJ (2009) Histone acetylation, VERNALIZATION INSENSITIVE 3, FLOWERING LOCUS C, and the vernalization response Mol Plant 2:724-737 doi:10.1093/mp/ssp021

Bonner M, Kmiec EB (2009) DNA breakage associated with targeted gene alteration directed by DNA oligonucleotides Mutat Res 669:85-94 doi:10.1016/j.mrfmmm.2009.05.004

Brachman EE, Kmiec EB (2005) Gene repair in mammalian cells is stimulated by the elongation of $S$ phase and transient stalling of replication forks DNA Repair (Amst) 4:445-457 doi:10.1016/j.dnarep.2004.11.007

Breyer D et al. (2009) Genetic modification through oligonucleotide-mediated mutagenesis. A GMO regulatory challenge? Environ Biosafety Res 8:57-64 doi:10.1051/ebr/2009007

Christensen AH, Quail PH (1996) Ubiquitin promoter-based vectors for high-level expression of selectable and/or screenable marker genes in monocotyledonous plants Transgenic Res 5:213-218

Chua YL, Watson LA, Gray JC (2003) The transcriptional enhancer of the pea plastocyanin gene associates with the nuclear matrix and regulates gene expression through histone acetylation Plant Cell 15:1468-1479

Delcuve GP, Khan DH, Davie JR (2012) Roles of histone deacetylases in epigenetic regulation: emerging paradigms from studies with inhibitors Clin Epigenetics 4:5 doi:10.1186/18687083-4-5

Dong C, Beetham P, Vincent K, Sharp P (2006) Oligonucleotide-directed gene repair in wheat using a transient plasmid gene repair assay system Plant Cell Rep 25:457-465 doi:10.1007/s00299005-0098-x

Earley KW, Shook MS, Brower-Toland B, Hicks L, Pikaard CS (2007) In vitro specificities of Arabidopsis co-activator histone acetyltransferases: implications for histone hyperacetylation in gene activation Plant J 52:615-626 doi:10.1111/j.1365-313X.2007.03264.x

Engstrom JU, Kmiec EB (2007) Manipulation of cell cycle progression can counteract the apparent loss of correction frequency following oligonucleotide-directed gene repair BMC Mol Biol 8:9 doi:10.1186/1471-2199-8-9

Engstrom JU, Kmiec EB (2008) DNA replication, cell cycle progression and the targeted gene repair reaction Cell Cycle 7:1402-1414 doi:10.4161/cc.7.10.5826

Fogarty RD, McKean SC, White PJ, Atley LM, Werther GA, Wraight CJ (2002) Sequence dependence of C5-propynyl-dU,dC-phosphorothioate oligonucleotide inhibition of the human IGF-I receptor: 
mRNA, protein, and cell growth Antisense Nucleic Acid Drug Dev 12:369-377 doi:10.1089/108729002321082447

Gilles AF, Averof M (2014) Functional genetics for all: engineered nucleases, CRISPR and the gene editing revolution Evodevo 5:43 doi:10.1186/2041-9139-5-43

Hu Y, Parekh-Olmedo H, Drury M, Skogen M, Kmiec EB (2005) Reaction parameters of targeted gene repair in mammalian cells Mol Biotechnol 29:197-210 doi:10.1385/MB:29:3:197

Huen MS, Li XT, Lu LY, Watt RM, Liu DP, Huang JD (2006) The involvement of replication in single stranded oligonucleotide-mediated gene repair Nucleic Acids Res 34:6183-6194 doi:10.1093/nar/gkl852

Huen MS, Lu LY, Liu DP, Huang JD (2007) Active transcription promotes single-stranded oligonucleotide mediated gene repair Biochem Biophys Res Commun 353:33-39 doi:10.1016/j.bbrc.2006.11.146

Kim H, Kim JS (2014) A guide to genome engineering with programmable nucleases Nat Rev Genet 15:321-334 doi:10.1038/nrg3686

Kochevenko A, Willmitzer L (2003) Chimeric RNA/DNA oligonucleotide-based site-specific modification of the tobacco acetolactate syntase gene Plant Physiol 132:174-184 doi:10.1104/pp.102.016857

Morocz S, Donn G, Nerneth J, Dudits D (1990) An improved system to obtain fertile regenerants via maize protoplasts isolated from a highly embryogenic suspension culture Theor Appl Genet 80:721-726 doi:10.1007/BF00224183

Okuzaki A, Toriyama K (2004) Chimeric RNA/DNA oligonucleotide-directed gene targeting in rice Plant Cell Rep 22:509-512 doi:10.1007/s00299-003-0698-2

Parekh-Olmedo H, Engstrom JU, Kmiec EB (2003) The effect of hydroxyurea and trichostatin a on targeted nucleotide exchange in yeast and Mammalian cells Ann N Y Acad Sci 1002:43-55

Paul AL, Ferl RJ (1993) Osmium tetroxide footprinting of a scaffold attachment region in the maize Adh1 promoter Plant Mol Biol 22:1145-1151

Paul AL, Ferl RJ (1998) Higher order chromatin structures in maize and Arabidopsis Plant Cell 10:1349-1359

Puchta H, Fauser F (2014) Synthetic nucleases for genome engineering in plants: prospects for a bright future Plant J 78:727-741 doi:10.1111/tpj.12338

Radecke S, Radecke F, Cathomen T, Schwarz K (2010) Zinc-finger nuclease-induced gene repair with oligodeoxynucleotides: wanted and unwanted target locus modifications Mol Ther 18:743753 doi:10.1038/mt.2009.304

Rivera-Torres N, Kmiec EB (2016) Genetic spell-checking: gene editing using single-stranded DNA oligonucleotides Plant Biotechnol J 14:463-470 doi:10.1111/pbi.12473

Ruiter R, van den Brande I, Stals E, Delaure S, Cornelissen M, D'Halluin K (2003) Spontaneous mutation frequency in plants obscures the effect of chimeraplasty Plant Mol Biol 53:675-689 doi:10.1023/B:PLAN.0000019111.96107.01

Ruxton GD (2006) The unequal variance t-test is an underused alternative to Student's t-test and the Mann-Whitney U test Behav Ecol 17:688-690 doi:10.1093/beheco/ark016

Sargent RG, Kim S, Gruenert DC (2011) Oligo/polynucleotide-based gene modification: strategies and therapeutic potential Oligonucleotides 21:55-75 doi:10.1089/oli.2010.0273

Sauer NJ et al. (2016a) Oligonucleotide-directed mutagenesis for precision gene editing Plant Biotechnol J 14:496-502 doi:10.1111/pbi.12496

Sauer NJ et al. (2016b) Oligonucleotide-Mediated Genome Editing Provides Precision and Function to Engineered Nucleases and Antibiotics in Plants Plant Physiol 170:1917-1928 doi:10.1104/pp.15.01696

Schrader C, Schielke A, Ellerbroek L, Johne R (2012) PCR inhibitors-occurrence, properties and removal Journal of applied microbiology 113:1014-1026 
Shu H, Gruissem W, Hennig L (2013) Measuring Arabidopsis chromatin accessibility using DNase Ipolymerase chain reaction and DNase I-chip assays Plant Physiol 162:1794-1801 doi:10.1104/pp.113.220400

Strouse B, Bialk P, Niamat RA, Rivera-Torres N, Kmiec EB (2014) Combinatorial gene editing in mammalian cells using ssODNs and TALENs Sci Rep 4:3791 doi:10.1038/srep03791

Svitashev S, Young JK, Schwartz C, Gao H, Falco SC, Cigan AM (2015) Targeted Mutagenesis, Precise Gene Editing, and Site-Specific Gene Insertion in Maize Using Cas9 and Guide RNA Plant Physiol 169:931-945 doi:10.1104/pp.15.00793

Voytas DF (2013) Plant genome engineering with sequence-specific nucleases Annu Rev Plant Biol 64:327-350 doi:10.1146/annurev-arplant-042811-105552

Voytas DF, Gao C (2014) Precision genome engineering and agriculture: opportunities and regulatory challenges PLoS Biol 12:e1001877 doi:10.1371/journal.pbio.1001877

Wang $M$ et al. (2015) Gene editing by co-transformation of TALEN and chimeric RNA/DNA oligonucleotides on the rice OsEPSPS gene and the inheritance of mutations PLoS One 10:e0122755 doi:10.1371/journal.pone.0122755

Wefers B, Meyer M, Ortiz O, Hrabe de Angelis M, Hansen J, Wurst W, Kuhn R (2013) Direct production of mouse disease models by embryo microinjection of TALENs and oligodeoxynucleotides Proc Natl Acad Sci U S A 110:3782-3787 doi:10.1073/pnas.1218721110

Yao Q, Cong L, Chang JL, Li KX, Yang GX, He GY (2006) Low copy number gene transfer and stable expression in a commercial wheat cultivar via particle bombardment J Exp Bot 57:3737-3746 doi:10.1093/jxb/erl145

Zhu T, Mettenburg K, Peterson DJ, Tagliani L, Baszczynski CL (2000) Engineering herbicide-resistant maize using chimeric RNA/DNA oligonucleotides Nat Biotechnol 18:555-558 doi:10.1038/75435

Zhu T, Peterson DJ, Tagliani L, St Clair G, Baszczynski CL, Bowen B (1999) Targeted manipulation of maize genes in vivo using chimeric RNA/DNA oligonucleotides Proc Natl Acad Sci U S A 96:8768-8773 


\section{Figures}
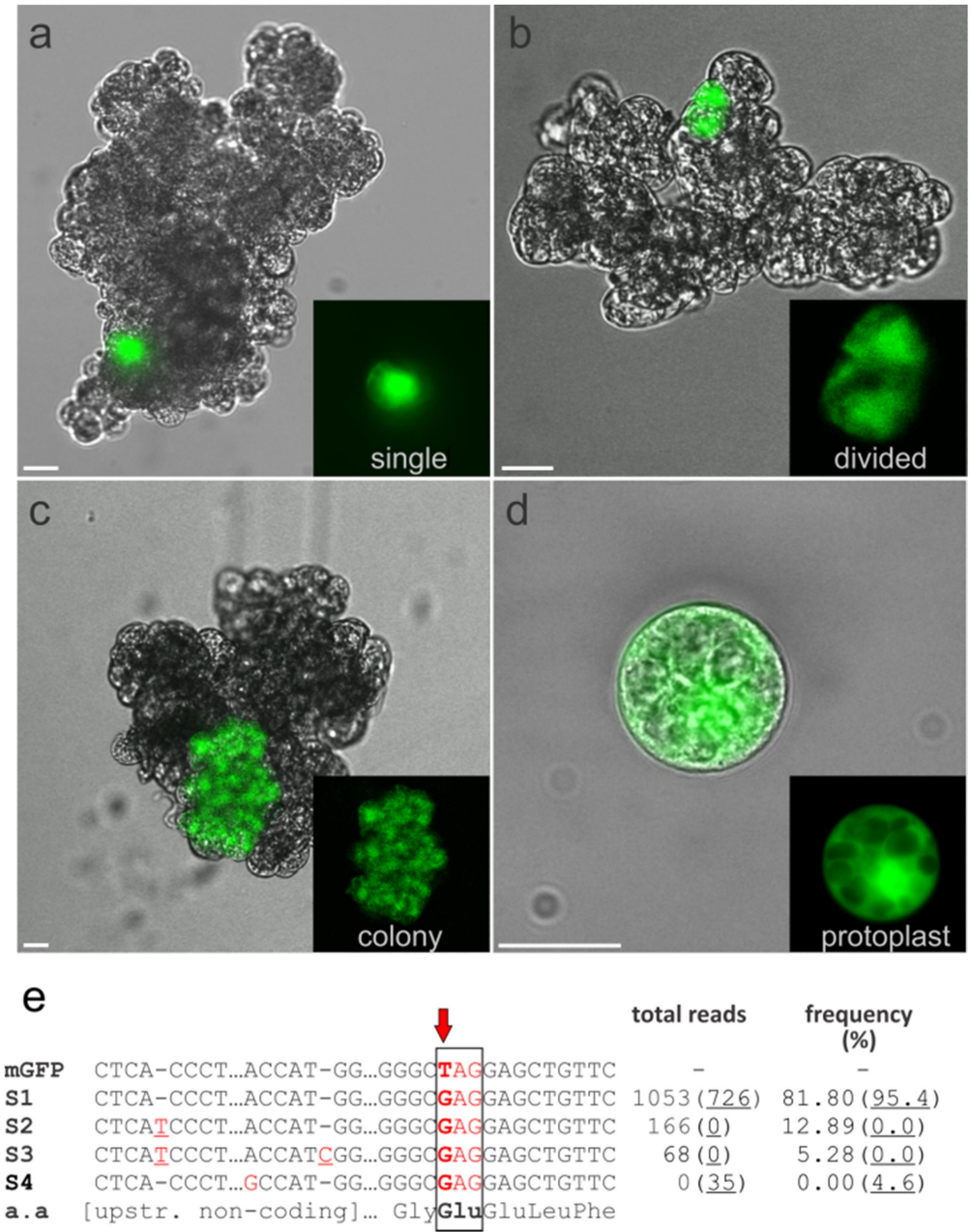

Fig 1 Nucleotide exchange resulted in the recovery of GFP phenotype after bombardment with synthetic DNA oligonucleotide (SDO ${ }^{\text {GFP }}$ )

Confocal microscopy images of a GFP expressing single cell (a), a divided cell (b), a multicellular cluster (c) and an isolated protoplast (d) imaged at 36h, 60h, 10 days and $48 \mathrm{~h}$ post-bombardment, respectively. Scale bars are $25 \mu \mathrm{m}$. Sequence confirmation of gene editing using ion torrent sequencing of untreated control and $10 \mathrm{mM}$ NaBt-treated samples (e). Red arrow indicates the position of critical T to G change converting STOP (TAG) codon in mGFP mutant cell line sequence to glutamic acid (GAG) codon. The total number of identical reads and their frequency (\%) are shown for sequences with more than 50 identical reads $(\mathrm{S} 1, \mathrm{~S} 2$ and $\mathrm{S} 3)$. NaBt frequencies are shown in parentheses. Underlined red colored nucleotides indicate low frequency sequence variations. Corresponding amino acids (a.a) of the edited region of GFP gene are shown and the critical glutamic acid residue is indicated in bold. 
a

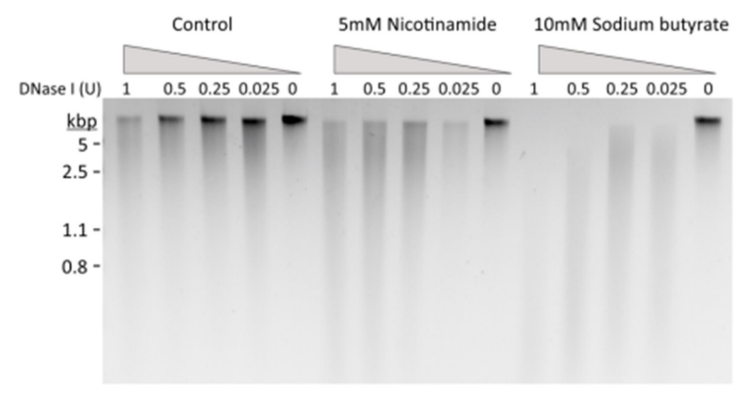

b
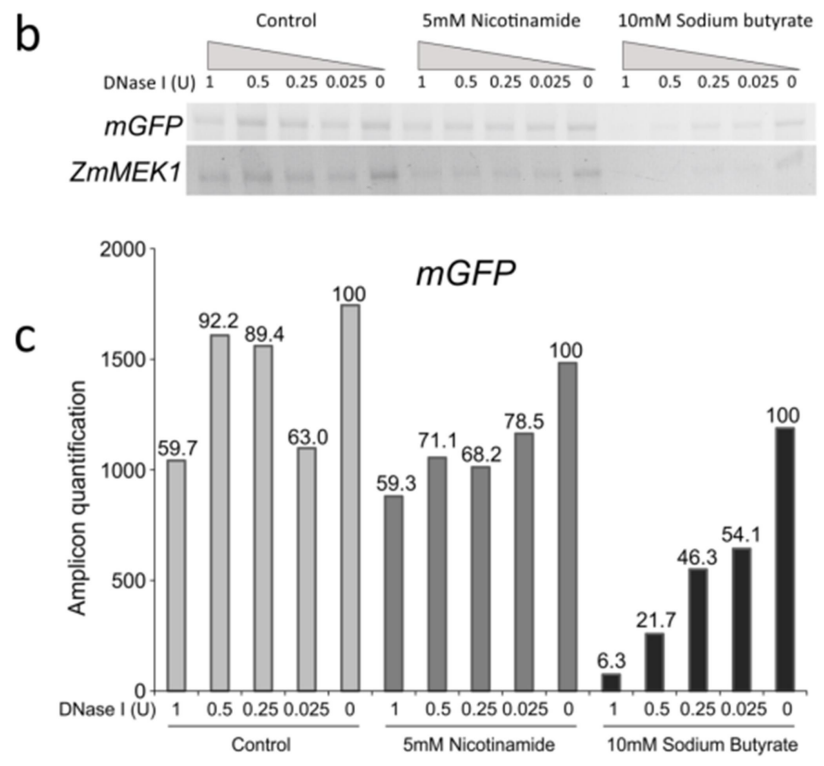

d

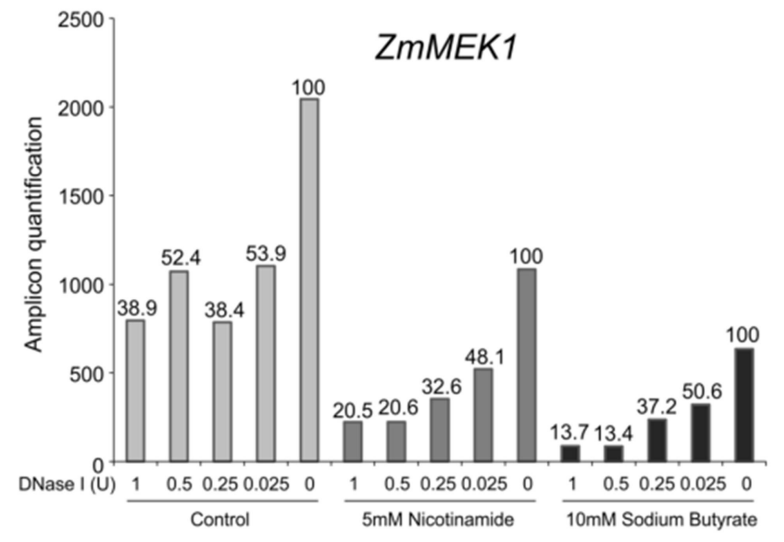

Fig. 2 Histone deacetylase inhibitors induce relaxed chromatin in cultured maize cells Increased DNase I sensitivity of chromatin from pretreated cells (5mM nicotinamide, $10 \mathrm{mM}$ sodium butyrate) and untreated (Control) cells as shown by resolved digestion products on agarose gel. In a concentration dependent way, all tested DNase I concentrations degraded the nuclear DNA isolated from the HDAC inhibitor-treated cells more efficiently as compared to control sample. (a) The units (U) of DNase I and molecular markers (kbp) are as shown. (b) PCR products showing detection of locus-specific chromatin structure by DNase I-Polymerase Chain Reaction Assay (Shu et al. 2013). The units (U) of DNase I and inhibitor concentrations $(\mathrm{mM})$ are as shown. Reduced amounts of PCR products from $m G F P$ and

ZmMEK1 genes indicate open chromatin at these loci. (c) Quantification of the PCR products for the $m G F P$ gene (in panel B) using gel intensity-based ImageJ Plot Analysis program. The units (U) of DNase I and inhibitor concentrations (mM) are as shown. (d) Quantification of the PCR products for the ZmMEK1 gene (in panel B) using gel intensity-based ImageJ Plot Analysis program. The units (U) of DNase I and inhibitor concentrations $(\mathrm{mM})$ are as shown. 

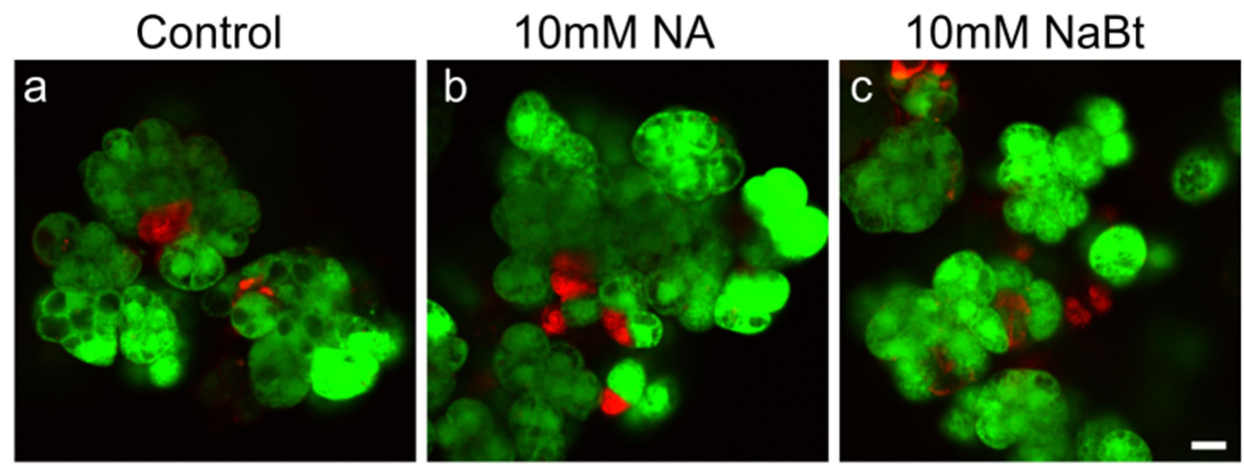

Fig. 3 Treatment with histone deacetylase inhibitors did not significantly interfere with the viability of maize cells

The dual-color viability assay using fluorescein diacetate (green, living cells) and propidium iodide (red, dead cells) are shown for untreated (Control) and 10mM inhibitor-treated maize cells. NA: Nicotinamide; NaBt: Sodium butyrate. Scale bar is $20 \mu \mathrm{m}$. 


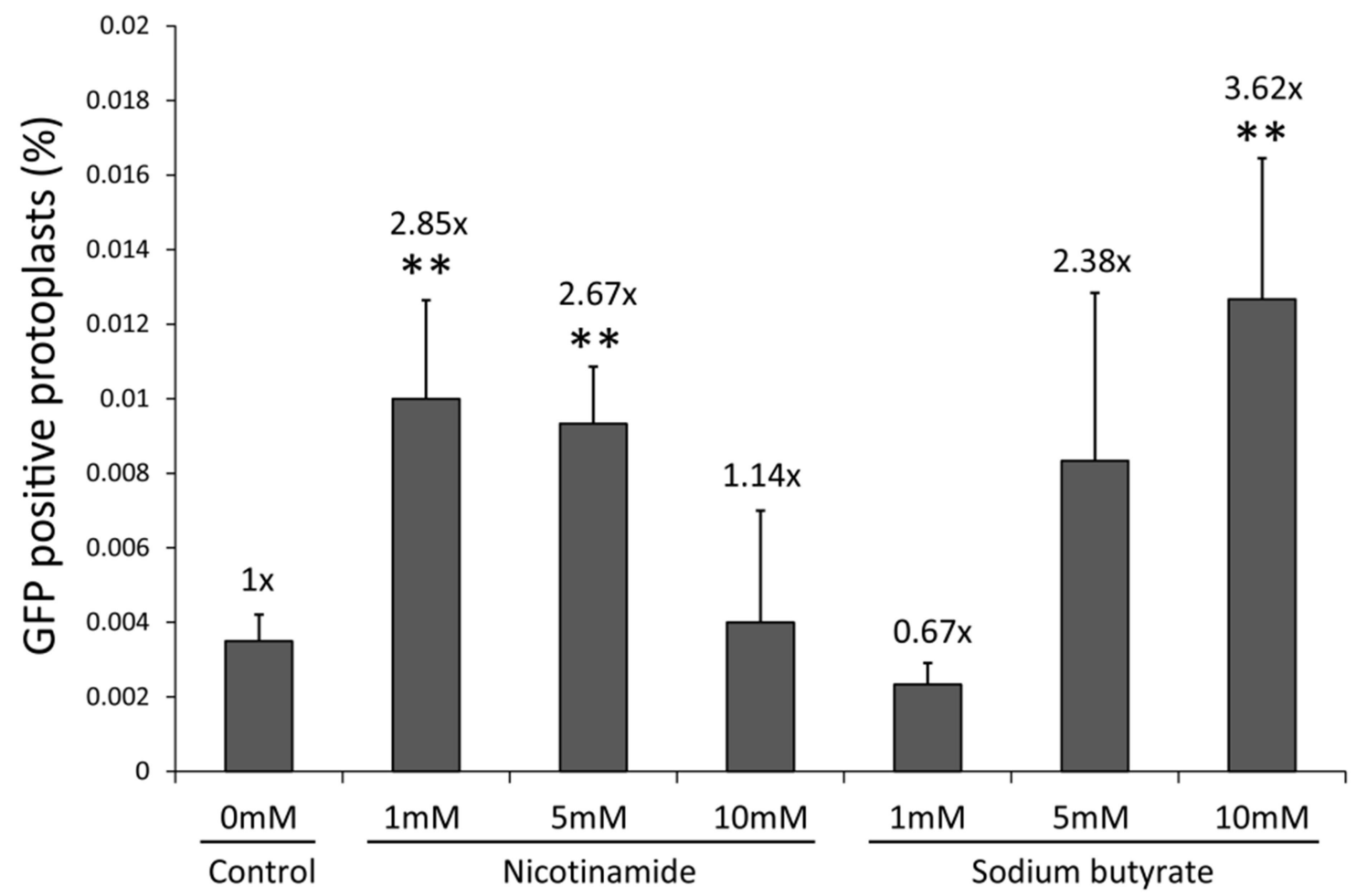

Fig. 4 Pre-treatment of maize cells with histone deacetylase inhibitors increased the frequency of GFP positive cells

The gene repair events were monitored by the restoration of mutated GFP gene by biolistic delivery of single-stranded DNA oligonucleotides into control and pretreated maize cells. Frequency of GFP positive protoplasts was quantified by flow cytometry and plotted. Double asterisks $(\mathrm{p}<0.05)$ indicate significant difference levels as compared to the untreated control sample using 95\% confidence level. Welch's t-test is used for pairwise comparisons. Numbers with muliplication symbol indicate how much more is the observed frequency of inhibitor-treated samples as compared to control sample (1x). Nicotinamide and sodium butyrate concentrations as well as mean values and standard deviations of three repetitions are as shown. 